PHYSICAL REVIEW D 87, 125002 (2013)

\title{
Chiral symmetry breaking in the pseudo-quantum electrodynamics
}

\author{
Van Sérgio Alves, ${ }^{*}$ Walace S. Elias, ${ }^{\dagger}$ and Leandro O. Nascimento \\ Faculdade de Física, Universidade Federal do Pará, Avenida Augusto Correa 01, 66075-110 Belém, Pará, Brazil \\ Vladimir Juričić ${ }^{\S}$ \\ Instituut-Lorentz for Theoretical Physics, Universiteit Leiden, P.O. Box 9506, 2300 RA Leiden, The Netherlands \\ Francisco Peñall \\ Departamento de Ciencias Físicas, Facultad de Ingeniería, Ciencias y Administracíon, Universidad de La Frontera, \\ Avenida Francisco Salazar 01145, Casilla 54-D, Teтuco, Chile
}

(Received 12 March 2013; published 3 June 2013)

\begin{abstract}
In this work, we study the chiral symmetry breaking in pseudo-quantum electrodynamics in $(2+1)$ dimensions, which is designed to reproduce a Coulomb potential for charged particles on a plane interacting via photons propagating in $(3+1)$ space-time dimensions and would be relevant for applications to condensed-matter systems. Using an ultraviolet cutoff in the momentum integrals, we show that there is a critical dimensionless coupling $\alpha_{c}=\pi / 4$ above which there is chiral symmetry breaking. In the case of the theory with $N$ massless fermions, we obtain a critical value of the number of fermion flavors, $N_{c}$, below which the chiral symmetry breaking occurs. Finally, we discuss the relevance of our results to graphene in the ultimate deep infrared regime where the Fermi velocity of the Dirac fermions approaches the velocity of light.
\end{abstract}

DOI: 10.1103/PhysRevD.87.125002

PACS numbers: 11.15.- q, 11.30.Rd, 73.22.Pr

\section{INTRODUCTION}

Gauge theories with $U(1)$ or $U(N)$ symmetries-such as the three-dimensional quantum electrodynamics $\left(\mathrm{QED}_{3}\right)$ - have many features similar to quantum chromodynamics, such as chiral symmetry breaking (CSB) and confinement [1-13]. The critical behavior of quantum electrodynamics in three as well as in four dimensions has been investigated close to the CSB transition, when fermions acquire dynamically generated mass. This is a nonperturbative effect and the coupled Schwinger-Dyson (SD) equations [14,15] for the fermion self-energy turned out to be appropriate to analyze the chiral phase transition [16].

In massless four-dimensional quantum electrodynamics $\left(\mathrm{QED}_{4}\right)$, the authors in Ref. [17], using the quenched approximation (without fermion loop corrections), obtained solutions of the SD equation for the fermion selfenergy. In Ref. [18] the theory with an ultraviolet (UV) cutoff $\Lambda$ was studied in the weak-coupling limit, and it was shown that for the values of the coupling constant $\alpha<\alpha_{c}=\pi / 3$, there exists no spontaneous chiral (or scale) symmetry breaking. The solutions of the SD equations for both weak and strong coupling were analyzed in detail in Ref. [19] where it has been found that in the massless case the spontaneous CSB occurs only if

\footnotetext{
*vansergi@ufpa.br

†walace@ufpa.br

†lonascimento@ufpa.br

\$uricic@lorentz.leidenuniv.nl

"fcampos@ufro.cl
}

$\alpha>\alpha_{c}=\pi / 3$, leading to generation of the fermion mass scale. By using a vertex ansatz proposed in Ref. [20], the authors in Ref. [21] have confirmed the existence of a critical coupling and have shown that the numerical changes are fairly small compared to the ladder approximation. Therefore, the ladder approximation is sufficient in order to obtain the critical coupling constant. Within the quenched ladder approximation with finite cutoff, considering the Landau gauge, this critical point $\alpha_{c}$ was interpreted by Miransky et al. [22] as a nontrivial ultraviolet fixed point defining the continuum limit of $\mathrm{QED}_{4}$, for which the corresponding scaling law for the dynamically generated mass is $\propto \Lambda \exp \left(-\pi / \sqrt{\alpha / \alpha_{c}-1}\right)$. Numerical simulations on the lattice in noncompact $\mathrm{QED}_{4}$ have confirmed such critical point [23]. In the unquenched approximation, taking into account the fermion-loop corrections in the vacuum polarization results in the changing of the Miransky scaling law to $\propto \Lambda \sqrt{\alpha-\alpha_{c}}$ [24]. Recently, the authors in Ref. [25] have shown, using a multiplicatively renormalizable photon propagator proposed in Ref. [26], that above a critical value of the fermion flavors $N_{c}$ chiral symmetry is restored and the scaling law is given by $\propto \Lambda \sqrt{N_{c}-N}$. As claimed by these authors, this critical number of fermion flavors is a consequence of the fact that there exists a critical value of the coupling constant above which the mass is dynamically generated.

In the context of massless $\mathrm{QED}_{3}$ with $N$ fermion flavors, in the lowest-order of $1 / N$ expansion, it is conjectured that there is a critical value for the number of flavors $\left(N_{c}=32 / \pi^{2} \approx 3.2\right)$ below which the CSB occurs $[1,5,27]$. In the framework of the numerical lattice 
simulations, it has been shown that $3<N_{c}<4$ [28]. Higher order corrections in $1 / N$ expansion suggest that the critical number of the fermion flavors does not qualitatively change from the lowest-order result [7]. Results obtained by using different types of ansatz for the vertex function, including those which satisfy the WardTakahashi identities $[10,12,20,29,30]$, also show that there is a critical number $N_{c}$ in $\mathrm{QED}_{3}$ below which CSB occurs and fermions acquire mass. However, the existence of this critical number has been criticized by some authors that suggest that chiral symmetry is dynamically broken for arbitrarily large $N$ in massless $\mathrm{QED}_{3}[8,12,31]$.

It should be noted that in $\mathrm{QED}_{3}$ all the above results were obtained using the fact that the electromagnetic field is confined to a plane. Although the electrons are confined to a plane, the electromagnetic field through which they interact may not be subjected to this constraint. This allows us to investigate more general physical situations, such as those where electrons are confined to a two-dimensional plane while the dynamical U(1) gauge field propagates into one extra spatial dimension. Thereby, the electrostatic potential between the electrons is Coulomb-like $(\propto 1 / r)$, and not logarithmic as is the case in $\mathrm{QED}_{3}$. Formally, the correct description of this system can be achieved in threedimensional Euclidean space-time by changing the usual Maxwell term $\sim F_{\mu \nu}^{2}$ by $\sim F_{\mu \nu}^{2} /(-\square)^{1 / 2}$, which reproduces the Coulomb interaction for two charged particles [32]. In Ref. [33], Marino obtained such an effective description of electrons moving in a plane, but interacting through an electromagnetic field embedded in a four-dimensional space-time, resulting in the nonlocal Maxwell-like term in the action. An equivalent version of this theory has been subsequently studied by Gonzalez et al. [34]. The quantization of such theory, called pseudo-quantum electrodynamics, has been studied in Ref. [35] where it has been shown that, despite the nonlocality of the Maxwell term, the causality is respected and its Green functions are well defined. In this scenario, the new Maxwell term makes the canonical dimension of the gauge field equal to one in mass units. Therefore, when the matter field is minimally coupled to the gauge field, the coupling constant becomes dimensionless. Thus, in the sense of power counting [14,36], three-dimensional pseudo-quantum electrodynamics $\left(\mathrm{PQED}_{3}\right)$ resembles more $\mathrm{QED}_{4}$ than $\mathrm{QED}_{3}$. On the other hand, since the charged fermionic matter is confined to the plane, we expect that the $\mathrm{PQED}_{3}$ retains some properties of $\mathrm{QED}_{3}$.

The purpose of this paper is to study the CSB in the $\mathrm{PQED}_{3}$ and its similarities with the criticality in both $\mathrm{QED}_{3}$ and $\mathrm{QED}_{4}$. Motivation for this work also stems, at least partially, from the relevance of $\mathrm{PQED}_{3}$ in condensedmatter physics. In particular, this theory is relevant for graphene which is a two-dimensional crystal of carbon atoms placed on a honeycomb lattice with massless linearly dispersing quasiparticles in the vicinity of the corners of the hexagonal Brillouin zone propagating with the Fermi velocity $v_{F} \sim c / 300$, where $c$ is the velocity of light [37]. Since the honeycomb lattice is bipartite and only two out of six corners of the hexagonal Brillouin zone are inequivalent, electrons in graphene, neglecting their spin, at low energies can be effectively described in terms of a four-component Dirac spinor with its dynamics given by the usual Dirac Lagrangian. Electrons confined in the graphene sheet, being charged, interact via the electromagnetic field which, however, propagates through threedimensional space. Therefore, the theory describing interacting Dirac fermions in graphene is $\mathrm{PQED}_{3}$-like with a fundamental difference: the Lorentz invariance is absent since $v_{F} \neq c$. Furthermore, since $v_{F} \ll c$, to study this system, it is thus enough to keep the static long-range Coulomb interaction, i.e., to neglect the retardation effects in the full theory. As the result, the Fermi velocity is renormalized, and flows logarithmically slowly to the velocity of light reached in the ultimate ultrarelativistic regime [34]. However, this regime is not experimentally reachable due to the logarithmically slow increase of the Fermi velocity. Interacting Dirac fermions in graphene are in this deep infrared regime described by $\mathrm{PQED}_{3}$ due to the restoration of the Lorentz symmetry as all velocities in the problem are then equal to the velocity of light. Therefore, the results of this paper may be relevant in this ultrarelativistic regime. Finally, let us mention that physics of graphene in this deep infrared regime has been recently studied in Refs. [38-40].

In order to study the CSB in $\mathrm{PQED}_{3}$ we adopt a strategy based on the coupled SD equations for the photon and fermion propagators which proved to be useful in the studies of the same phenomenon in $\mathrm{QED}_{3}$ and $\mathrm{QED}_{4}$. The paper is organized as follows. In Sec. II we present the model and make a connection to the physical situation in graphene. In Sec. III we set up the SD equations for the photon and fermion propagators and we present the truncation scheme used in this paper which we wish to examine. In Sec. IV we investigate the analytical solutions of the integral equation for the mass function of fermions in its linearized form. In Sec. V we examine the influence of vacuum polarization on the CSB using the $N$ massless fermion flavors version and adopting a $1 / N$ expansion. We summarize our results in Sec. VI. In Appendices A and $\mathrm{B}$ we numerically solve the nonlinear integral equation for the dynamically generated mass and the integral equation for the wave function renormalization, respectively, confirming the analytical results obtained in Secs. IV and V.

\section{THE MODEL}

Let us start by considering theory of the massive fermions given by the following Lagrangian in threedimensional Euclidean space-time 


$$
\begin{aligned}
\mathcal{L}= & \frac{1}{4} \frac{F_{\mu \nu} F^{\mu \nu}}{(-\square)^{1 / 2}}+\bar{\psi}\left(i \gamma^{\mu} \partial_{\mu}+m+\frac{e}{2} \gamma^{\mu} A_{\mu}\right) \psi \\
& -\frac{\xi}{2} A_{\mu} \frac{\partial^{\mu} \partial^{\nu}}{(-\square)^{1 / 2}} A_{\nu},
\end{aligned}
$$

where $\square$ is the d'Alembertian operator, $m$ is the bare fermion mass which explicitly breaks both chiral and scale symmetry, and $e$ is the unrenormalized (bare) coupling constant. The last term is the gauge fixing. In the above Lagrangian, we have set both the velocity of the fermions, $v_{F}$, recovered in the above Lagrangian by substituting $\gamma^{\mu} \partial_{\mu} \equiv \gamma^{0} \partial_{0}+\gamma^{i} \partial_{i} \rightarrow \gamma^{0} \partial_{0}+v_{F} \gamma^{i} \partial_{i}$, and the velocity of the gauge field (the velocity of light), $c$, equal to one. However, in general, these two velocities need not be equal. In particular, in graphene, the Fermi velocity of the low-energy Dirac quasiparticles is $v_{F} \sim c / 300$, and as the result the pseudo-Lorentz invariance of the graphene's noninteracting Dirac Lagrangian is broken. The mass $m$ in Lagrangian (1), when a suitable representation of the $\gamma$ matrices is chosen, describes a possible charge-densitywave gap in the spectrum of electrons on the honeycomb lattice which breaks the sublattice symmetry [41]. Other relativistically invariant masses, neglecting possible superconducting instability, correspond to breaking of translational-lattice, time-reversal, or spin-rotational symmetry of the electrons on the graphene's honeycomb lattice [42]. Even more possibilities for the relativistic mass gaps arise when the superconducting instability is taken into account $[43,44]$.

The Lagrangian (1) was proposed by Marino [33] to describe the effective electromagnetic interaction for particles confined to a plane. It is obtained by dimensional reduction of $\mathrm{QED}_{4}$ (spinor or scalar) considering that the current satisfies the condition $J_{3+1}^{\mu}\left(x^{0}, x^{1}, x^{2}, x^{3}\right)=$ $J^{\mu}\left(x^{0}, x^{1}, x^{2}\right) \delta\left(x^{3}\right)$ for $\mu=0,1,2$ and it is null for $\mu=$ 3 which takes into account that the fermions are confined to a plane. In fact, defining the functional generator to the Green function of the gauge field, we obtain

$$
\begin{aligned}
Z\left[J^{\mu}\right] & =\int D A_{\mu} e^{-\int d^{3} x\left[\frac{\left[{ }^{F} \mu \nu F^{\mu \nu}\right.}{(-\square)^{1 / 2}}-\frac{\xi}{2} A_{\mu} \frac{\partial^{\mu} \partial^{\nu}}{(-\square)^{1 / 2}} A_{\nu}\right]} e^{-\frac{e}{2} \int d^{3} x J^{\mu} A_{\mu}} \\
& =\int D A_{\mu} e^{-\int d^{3} x\left[\frac{1}{2} A_{\mu} \mathcal{O}^{\mu \nu} A_{\nu}+\frac{e}{2} J^{\mu} A_{\mu}\right]} \\
& =e^{\frac{e^{2}}{8} \int d^{3} x J^{\mu} \mathcal{O}_{\mu \nu}^{-1} J^{\nu}},
\end{aligned}
$$

where $J^{\mu}=\bar{\psi} \gamma^{\mu} \psi$, and

$$
\mathcal{O}^{\mu \nu}=-\delta^{\mu \nu} \frac{\square}{(-\square)^{1 / 2}}+(1-\xi) \frac{\partial^{\mu} \partial^{\nu}}{(-\square)^{1 / 2}} .
$$

Since the current is conserved, Eq. (2) leads, together with the Dirac Lagrangian, to the following effective theory

$$
\mathcal{L}_{\text {eff }}=-\frac{e^{2}}{8} \bar{\psi} \gamma^{\mu} \psi \frac{1}{(-\square)^{1 / 2}} \bar{\psi} \gamma_{\mu} \psi+\bar{\psi} i \gamma^{\mu} \partial_{\mu} \psi,
$$

which is the same effective theory obtained by dimensional reduction of $\mathrm{QED}_{4}$ when the matter is confined to the $x_{1}-x_{2}$ plane, after the gauge field has been integrated out [33]. Therefore, this theory has similarities with both $\mathrm{QED}_{3}$ (particles moving on the plane with the gauge field confined to the same plane) and $\mathrm{QED}_{4}$ (both the fermions and the gauge field "live" in four-dimensional space-time). Furthermore, the interaction above is dynamical and it fully incorporates retardation effects, since this theory has Lorentz invariance. On the other hand, when the Fermi velocity $v_{F}$ is explicitly recovered in Lagrangian (4), and after taking the limit $v_{F} \ll c$, the static long-range Coulomb interaction with a $1 / r$-tale is recovered.

In this paper, we are interested in CSB in three-dimensional space-time, and use a four-dimensional representation of gamma matrices [45,46]. As the coupling constant $e$ in Eq. (1) is dimensionless, the theory is renormalizable in the usual power counting sense, unlike the usual quantum electrodynamics in three space-time dimensions which is superrenormalizable and ultraviolet finite. The Feynman rules for the model are obtained as usual. The interaction vertex is given by $e \gamma^{\mu} / 2$ and the inverse bare fermion propagator is

$$
S_{0 F}^{-1}(p)=-\gamma^{\mu} p_{\mu}+m .
$$

The bare gauge field propagator can be obtained from (3), and in momentum space reads

$$
\Delta_{0 \mu \nu}(p)=\frac{1}{\left(p^{2}\right)^{1 / 2}}\left[\delta_{\mu \nu}-\left(1-\frac{1}{\xi}\right) \frac{p_{\mu} p_{\nu}}{p^{2}}\right] .
$$

As we can observe, the propagator above has a better infrared behavior than in the $\mathrm{QED}_{4}$ theory. Moreover, the fact that the propagator behaves as $1 / p$ will lead to a much simpler kernel than $\mathrm{QED}_{3}$ or $\mathrm{QED}_{4}$, when we consider the vacuum polarization, as discussed in Sec. V.

\section{THE COUPLED SD EQUATIONS}

The inverse of fermion and photon propagators read

$$
S_{F}^{-1}(p)=S_{0 F}^{-1}(p)-\Xi(p)
$$

and

$$
\Delta_{\mu \nu}^{-1}(p)=\Delta_{0 \mu \nu}^{-1}-\Pi_{\mu \nu}(p)
$$

with the corresponding diagrammatic representation shown in Figs. 1 and 2, respectively. The self-energies are

$$
\Xi(p)=\frac{e^{2}}{4} \int \frac{d^{3} k}{(2 \pi)^{3}} \gamma^{\mu} S_{F}(k) \Gamma^{\nu}(k, p) \Delta_{\mu \nu}(p-k)
$$

and

$$
\Pi^{\mu \nu}(p)=-\frac{e^{2}}{4} \int \frac{d^{3} k}{(2 \pi)^{3}} \operatorname{Tr}\left[\gamma^{\mu} S_{F}(k+p) \Gamma^{\nu}(k, p) S_{F}(k)\right],
$$

representing the coupled SD equations for the fermion and for the photon. The factor $(-1)$ in Eq. (10) arises from the 


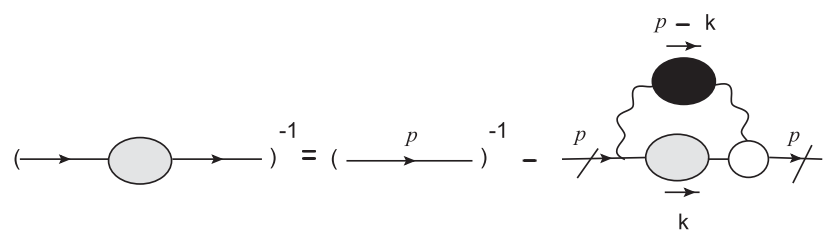

FIG. 1. The fermion SD equation. Filled dots indicate full propagators and vertex. The second term on the right-hand side represents the fermion self-energy $\Xi(p)$.

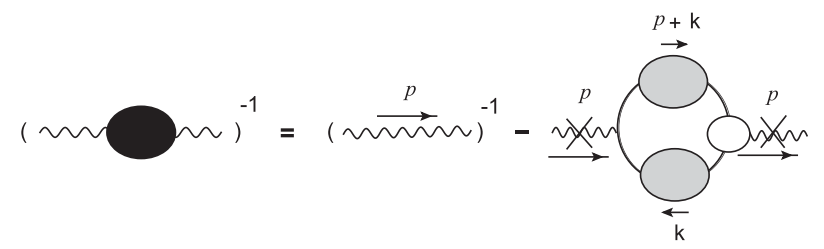

FIG. 2. The photon SD equation. Filled dots indicate full propagators and vertex. The second term on the right-hand side represents the photon self-energy $\Pi^{\mu \nu}(p)$.

fermion loop in the usual way. In the above expressions, $S_{F}, \Delta_{\mu \nu}$, and $\Gamma^{\nu}$ mean full propagators and vertex, respectively, and $\operatorname{Tr}$ denotes trace over the spinor indices.

Equations (9) and (10) form a set of complicated coupled nonlinear integral equations and this calls for an approximation to treat this problem. The most common approximation used in literature is the ladder approximation for vertex, also called rainbow approximation, that uses a bare vertex for the boson-fermion coupling, i.e., $\Gamma^{\nu}(k, p)=\gamma^{\nu}$. When this approximation is combined with the quenched approximation, i.e., neglecting fermion-loop contributions to the vacuum polarization, meaning $\Delta_{\mu \nu}(p)=\Delta_{0 \mu \nu}(p)$, Eqs. (9) and (10) decouple and can be studied separately. In this situation we can write the inverse of the full fermion propagator as

$S_{F}^{-1}(p)=S_{0 F}^{-1}(p)-\pi \alpha \int \frac{d^{3} k}{(2 \pi)^{3}} \gamma^{\mu} S_{F}(k) \gamma^{\nu} \Delta_{0 \mu \nu}(p-k)$,

where we have defined $e^{2}=4 \pi \alpha$ as in $\mathrm{QED}_{4}$.

In Euclidean space we can write the inverse fermion propagator as

$$
S_{F}^{-1}(p)=-p_{\mu} \gamma^{\mu} A(p)+\Sigma(p),
$$

where $A(p)$ is the wave function renormalization and $\Sigma(p)$ is the mass function which leads to dynamically generated mass for fermions. Substituting Eq. (12) into Eq. (11), we get

$$
\begin{aligned}
& -p_{\alpha} \gamma^{\alpha} A(p)+\Sigma(p) \\
& =-p_{\alpha} \gamma^{\alpha}+m-\pi \alpha \int \frac{d^{3} k}{(2 \pi)^{3}} \gamma^{\mu} \\
& \quad \times\left[\frac{1}{-k_{\beta} \gamma^{\beta} A(k)+\Sigma(k)}\right] \gamma^{\nu} \Delta_{0 \mu \nu}(p-k) .
\end{aligned}
$$

From the equation above, we can obtain expressions for $A(p)$ and $\Sigma(p)$ using simple trace operators over the $\gamma$ matrices. Multiplying both sides of Eq. (13) by $\gamma_{\mu} p^{\mu}$ and then taking the trace on both sides, we conclude that, perturbatively, $A(p)=1+\mathcal{O}(\alpha)$ (see Appendix B). On the other hand, taking the trace on both sides in Eq. (13), using the identities in Ref. [47], we obtain the following equation for $\Sigma(p)$ :

$$
\Sigma(p)=m+\pi \alpha \int \frac{d^{3} k}{(2 \pi)^{3}} \frac{\Sigma(k) \delta_{\mu \nu} \Delta_{0 \mu \nu}(p-k)}{A^{2}(k) k^{2}+\Sigma^{2}(k)} .
$$

Using the Landau gauge $\xi \rightarrow \infty$ and carrying out the angular integration, Eq. (14) takes the form

$$
\Sigma(p)=m+\frac{\alpha}{2 \pi p} \int_{0}^{\infty} d k \frac{k \Sigma(k)(|p+k|-|p-k|)}{A^{2}(k) k^{2}+\Sigma^{2}(k)} .
$$

Although the inclusion of higher order terms in $A(p)$ is essential to recover the gauge invariance [29], when we use that in leading order $A(p) \approx 1$, the qualitative results for $\Sigma(p)$ as a function of the coupling strength are not changed [4]. Provided that we are interested in CSB, we will consider from now on $m=0$, which is also motivated by the physical situation in graphene. In this case, since the theory does not have an intrinsic mass scale, it is more convenient to use a finite Euclidean ultraviolet cutoff $\Lambda$ to set our scale to study CSB $[22,48]$. We can always set $\Lambda=\infty$ at any point in our calculation. Using this and breaking the momentum integration into two regions, Eq. (15) becomes

$$
\Sigma(p)=\frac{\alpha}{\pi p}\left\{\int_{0}^{p} \frac{k^{2} \Sigma(k)}{k^{2}+\Sigma^{2}(k)} d k+p \int_{p}^{\Lambda} \frac{k \Sigma(k)}{k^{2}+\Sigma^{2}(k)} d k\right\} .
$$

The integral above can be conveniently transformed into an ordinary differential equation with two appropriate boundary conditions. Indeed, if we differentiate Eq. (16) with respect to $p$ we obtain

$$
\Sigma^{\prime}(p)=-\frac{\alpha}{\pi p^{2}} \int_{0}^{p} \frac{k^{2} \Sigma(k)}{k^{2}+\Sigma^{2}(k)} d k
$$

Multiplying by $p^{2}$ and differentiating once again we find

$$
p^{2} \Sigma^{\prime \prime}(p)+2 p \Sigma^{\prime}(p)+\frac{\alpha}{\pi} \frac{p^{2} \Sigma(p)}{p^{2}+\Sigma^{2}(p)}=0 .
$$

Using Eqs. (16) and (17), we find that

$$
\lim _{p \rightarrow \Lambda}\left(p \frac{d \Sigma(p)}{d p}+\Sigma(p)\right)=0
$$

and

$$
\lim _{p \rightarrow 0} p^{2} \frac{d \Sigma(p)}{d p}=0
$$

which represent the ultraviolet (UV) and infrared (IR) boundary conditions, respectively. The existence of a nontrivial 
solution for the dynamical fermion mass given by Eq. (18) indicate the existence of CSB.

We must use some additional conditions to obtain analytical solutions for Eq. (18). We will use the same approach applied to differential equations to study CSB in $\mathrm{QED}_{3}$ and $\mathrm{QED}_{4}$ given by $p \gg \Sigma(p)$. This approach was confirmed for both theories in many different contexts $[1,3-5,12,49]$. Furthermore, this approach is in agreement with the integral equation (17) for the first derivative of $\Sigma(p)$. Indeed $\Sigma^{\prime}(p) \leq 0$, then when $p$ increases $\Sigma(p)$ decreases, so we expect that $\Sigma(p \rightarrow \infty)=0$.

For small momenta $p \approx 0$, where the nonlinear term is more relevant, we show in Appendix A that for the behavior of the numerical solution, this nonlinearity is not important. Therefore, the analytical solution will behave almost identically as the numerical solution, since the nonlinear term $p^{2}+\Sigma^{2}(p) \approx p^{2}+\Sigma^{2}(0)$ for all external momenta. Indeed, the authors in Refs. [12,50] adopted this condition to linearize the differential equation in $\mathrm{QED}_{3}$ and they have not found any change in the critical behavior of the theory, in comparison with the approximation $p \gg \Sigma(p)$. In the next section we apply these approaches to $\mathrm{PQED}_{3}$.

\section{ANALYTICAL SOLUTION FOR $\Sigma(p)$}

\section{A. $p \gg \Sigma(p)$ approximation}

Using $p \gg \Sigma(p)$ to study the critical behavior of $\Sigma(p)$, the Eq. (18) takes the simple linear form

$$
\frac{d}{d p}\left(p^{2} \frac{d \Sigma(p)}{d p}\right)+\frac{\alpha}{\pi} \Sigma(p)=0,
$$

which has the following solution:

$$
\Sigma(p)=\Sigma_{1}(p)+\Sigma_{2}(p)=B_{1} p^{-\frac{\lambda}{2}}+B_{2} p^{-1+\frac{\lambda}{2}},
$$

where $\lambda=1-\sqrt{1-\alpha / \alpha_{c}}$, with the critical coupling $\alpha_{c}=\pi / 4$. The arbitrary constants $B_{1}$ and $B_{2}$ have dimension, respectively, given by $1+\frac{\lambda}{2}$ and $2-\frac{\lambda}{2}$, in mass units. In this linear regime we can analyze two relevant limits concerning the cutoff $\Lambda$.

\section{The limit $\Lambda \rightarrow \infty$}

It should be noted that in the limit the solutions in Eq. (22) satisfy both IR and UV boundary conditions for any range of $\alpha$. Therefore, CSB occurs in $\mathrm{PQED}_{3}$ within the rainbow-quenched approximation for all values of the coupling constant in this limit of the cutoff which is comparable to the result for $\mathrm{QED}_{4}$ in Ref. [51].

\section{Finite cutoff $\Lambda$}

Motivated by the physical situation in the electron systems on a lattice, as well as by the possible relevance of our study for some of these systems, we now take the cutoff $\Lambda$ to be finite. In this case, it is more convenient to solve
Eq. (21) and use the IR boundary condition first. Then, we use the UV boundary condition to determine whether or not this is a nontrivial solution of the SD equation. For the case in which $\alpha<\alpha_{c}$ we have $0<\lambda<1$ and the two solutions do not satisfy the UV boundary condition. The only analytical solution for this case is $\Sigma(p)=0$ and there is no CSB.

For $\alpha>\alpha_{c}, \lambda$ is complex and Eq. (21) admits the following oscillatory solution:

$$
\begin{aligned}
\Sigma(p) & =\frac{1}{\sqrt{p}}\left[C_{1} \cos (\beta \log p)+i C_{2} \sin (\beta \log p)\right] \\
& =\frac{D}{\sqrt{p}} \sin \left[\beta\left(\log \frac{p}{\bar{\Sigma}(0)}+\delta\right)\right]
\end{aligned}
$$

where $2 \beta=\sqrt{\alpha / \alpha_{c}-1}>0, D=\sqrt{C_{1}^{2}-C_{2}^{2}}$ with $C_{1}=$ $B_{1}+B_{2}, C_{2}=B_{2}-B_{1}$, and $\beta \delta=\arctan \left(-i C_{1} / C_{2}\right)$ is a phase. We have introduced a logarithmic scale factor $\bar{\Sigma}(0)$ in the last line of the Eq. (23). This scale factor can be obtained imposing the UV boundary condition, which gives, for $\alpha \rightarrow \alpha_{c}$,

$$
\bar{\Sigma}(0)=\Lambda e^{2+\delta} \exp \left[-\frac{2 n \pi}{\sqrt{\frac{\alpha}{\alpha_{c}}-1}}\right],
$$

where $n=0,1,2, \ldots$. Therefore, we can conclude that chiral symmetry may be dynamically broken in the rainbow-quenched $\mathrm{PQED}_{3}$ if the coupling exceeds $\alpha_{c}$ for finite $\Lambda$. The scale parameter exhibits a phase transition as $\alpha \rightarrow \alpha_{c}$ and obeys the Miransky scaling law [22] which is a well-known result in the context of $\mathrm{QED}_{4}[16,22,52]$. In order to check these analytical solutions, we numerically solved Eq. (15) in Appendix A which indeed confirms the analytical solutions obtained in this section.

\section{B. $p^{2}+\Sigma^{2}(p) \approx p^{2}+\Sigma^{2}(0)$ approximation}

By using the approach proposed by Refs. [12,50], $p^{2}+\Sigma^{2}(p) \approx p^{2}+\Sigma^{2}(0)$, we will show that the critical behavior obtained from the linearization $p \gg \Sigma(p)$ does not change. Moreover, the solution obtained from this approach is compatible with the Miransky scaling law which we found earlier. First, notice that Eq. (18) admits the following solution:

$$
\Sigma(p)=\Sigma(0)_{2} F_{1}\left(\gamma, \gamma^{*}, \frac{3}{2},-\frac{p^{2}}{\Sigma^{2}(0)}\right),
$$

where

$$
\gamma=\frac{1}{4}-\frac{i}{4} \sqrt{\frac{\alpha}{\alpha_{c}}-1},
$$

and ${ }_{2} F_{1}$ is the confluent hypergeometric function, which satisfies the IR boundary condition for all values of $\alpha$. For $\Lambda \rightarrow \infty$, the UV boundary condition (19) is also satisfied, then the solution (25) implies CSB for all values of the 
coupling constant in the limit $\Lambda \rightarrow \infty$, confirming the results obtained previously.

In the case with $\Lambda$ finite it is not easy at first to analyse the UV boundary condition for all values of the coupling constant. However, the solution above clearly has a different behavior at $\alpha<\alpha_{c}=\pi / 4$ ( $\gamma$ is real) or $\alpha>\alpha_{c}=$ $\pi / 4$ ( $\gamma$ is complex). Indeed, using $\Lambda=10$ for convenience, and therefore measuring the momentum and energy in units of $\Lambda / 10$, as well as $\alpha=0.9 \alpha_{c}$, we obtain that $\Sigma(0) \approx 10^{-9}$, which is negligible. Therefore, the chiral symmetry can be taken as exact with a large precision which we also verified using different values of the UV cutoff.

Using $\alpha=1.5 \alpha_{c}$ ( $\gamma$ is complex) and the value of the cutoff as before, then imposing the UV boundary conditions, we find $\operatorname{Re}\{\Sigma(0)\}=0.033$ (considering just the real part of the solution). The term $\Sigma(0)$ increases by a factor of $10^{7}$ in the units of $\Lambda / 10$, thus, we conclude that CSB occurs only for $\alpha>\alpha_{c}=\pi / 4$. Keeping $\alpha=1.5 \alpha_{c}$ and changing the UV cutoff, it is possible to obtain the exact solution $\operatorname{Re}\{\Sigma(0)\}=0.0033 \Lambda$. Keeping $\Lambda=10$ and changing the coupling constant $\alpha$, it is possible to show that the numerical solutions behave like $\operatorname{Re} \Sigma(0)=$ $e^{2+\delta} \Lambda e^{-C / \sqrt{\alpha / \alpha_{c}-1}}$ with $\delta=-1.22$ and $C=-3.99$. The mass scale $\operatorname{Re} \Sigma(0)$ obeys the Miransky scaling law, like the parameter $\bar{\Sigma}(0)$ obtained in the last section. Furthermore, the critical coupling constant is exactly the same $\alpha_{c}=\pi / 4$.

According to Refs. [12,50], this approximation is adequate for both small and large momenta, because for small momenta $\Sigma(p)$ is almost constant, and for large momenta, both $\Sigma^{2}(p)$ and $\Sigma^{2}(0)$ are negligible with respect to $p^{2}$. Therefore, as we obtain the same critical behavior and similar mass scale using different approaches, according to the results of this section, in the next section, where we study CSB in terms of the number of the fermion flavors, we will adopt the simplest approach $p \gg \Sigma(p)$.

\section{THE CASE OF $N$ MASSLESS FERMION FLAVORS}

Now we will examine the influence of vacuum polarization on the CSB in the model with $N$ massless fermion flavors. For this purpose, we will use the $1 / N$ approximation and extend the Lagrangian (1) to $N$ massless fermions

$$
\begin{aligned}
\mathcal{L}= & \frac{1}{4} \frac{F_{\mu \nu} F^{\mu \nu}}{(-\square)^{1 / 2}}+\sum_{a=1}^{N} \bar{\psi}^{a}\left(i \gamma^{\mu} \partial_{\mu}+\frac{e}{2} \gamma^{\mu} A_{\mu}\right) \psi^{a} \\
& +\frac{\xi}{2} A_{\mu} \frac{\partial^{\mu} \partial^{\nu}}{(-\square)^{1 / 2}} A_{\nu} .
\end{aligned}
$$

We have now two independent parameters: the coupling constant $e$ and the number of fermion flavors $N$. This will allow us to make a comparison with the criticality of
$\mathrm{QED}_{3}$, as we shall see. Therefore, we assume that if the mass generation occurs, it must be for small $N$.

The vacuum polarization tensor in the limit of vanishing fermion mass reads

$$
\Pi^{\mu \nu}(p)=\Pi\left(p^{2}\right)\left(\delta^{\mu \nu}-\frac{p^{\mu} p^{\nu}}{p^{2}}\right)
$$

with $\Pi\left(p^{2}\right)=(g / 8) \sqrt{p^{2}}[2,3]$. Thus, the full photon propagator is

$$
\Delta^{\mu \nu}(p)=\frac{1}{\sqrt{p^{2}}\left(1+\frac{g}{8}\right)}\left(\delta^{\mu \nu}-\frac{p^{\mu} p^{\nu}}{p^{2}}\right),
$$

which shows that the vacuum polarization effect does not change the infrared and ultraviolet behavior of the gauge field propagator, as expected since the propagator is nonanalytic in the momentum [53]. Replacing $\pi \alpha \rightarrow g / N$ and $\Delta_{0 \mu \nu} \rightarrow \Delta_{\mu \nu}$ in Eq. (14), using the leading order for the vertex and putting $A(p) \approx 1$ (see Appendix B), we obtain a differential equation similar to Eq. (18). This differential equation satisfies the same boundary conditions as Eq. (19) and (20), and therefore the conclusions obtained in the previous case (rainbow-quenched approximation) can be easily extended to the rainbow unquenched approximation.

The solution of the linearized differential equation (18) is given by Eq. (22) with

$$
\lambda=1-\sqrt{1-\frac{4 g}{N \pi^{2}\left(1+\frac{g}{8}\right)}} .
$$

As now we have two independent dimensionless parameters $N$ and $g$, we have freedom in choosing the parameter to be used to study the criticality. In this case, it is natural to choose the number of fermion flavors for which, from the above equation, we obtain the critical number for the restoration of the chiral symmetry

$$
N_{c}=\frac{4 g}{\pi^{2}\left(1+\frac{g}{8}\right)},
$$

which is a result different than in $\mathrm{QED}_{3}$ and $\mathrm{QED}_{4}$. Furthermore, it is interesting to observe that $N_{c}$ increases slowly with $g$. Its maximum value is given by $N_{c}^{\max }=$ $32 / \pi^{2}$, for $g \rightarrow \infty$, which is also the critical value obtained in $\mathrm{QED}_{3}$ [1,5]. Since in the limit $\Lambda \rightarrow \infty$ CSB occurs for any number of fermion flavors similar as in the rainbowquenched approximation, in the following we will investigate the case of a finite cutoff.

\section{A. Finite cutoff $\boldsymbol{\Lambda}$}

For $N>N_{c}$ we have nonoscillatory solutions that satisfy the IR condition, but do not satisfy the UV boundary condition. Thus, as before, the only analytical solution is $\Sigma(p)=0$ and therefore there is no CSB.

For $N<N_{c}$ the solution that satisfies the UV boundary condition is given by the equation 


$$
\Sigma(p)=\frac{\tilde{D}}{\sqrt{p}} \sin \left[\frac{1}{2} \sqrt{\frac{N_{c}}{N}-1}\left(\log \frac{p}{\tilde{\Sigma}(0)}+\tilde{\delta}\right)\right],
$$

where $\tilde{D}$ and $\tilde{\delta}$ are constants and for $N \rightarrow N_{c}$ the mass scale is

$$
\tilde{\Sigma}(0)=\Lambda e^{2+\tilde{\delta}} \exp \left[-\frac{2 n \pi}{\sqrt{\frac{N_{c}}{N}-1}}\right],
$$

which also exhibits Miransky scaling law as the rainbowquenched approximation. Therefore, we can conclude that within this approximation there is a critical number of Dirac fermion flavors $N_{c}$ given by Eq. (31) below which the chiral symmetry breaking takes place. On the other hand, above this critical number of fermion flavors, the chiral symmetry is restored.

\section{FINAL REMARKS}

In this paper, we have studied the chiral symmetry breaking in pseudo-quantum electrodynamics in $(2+1)$ dimensions. We obtained analytical solutions of Schwinger-Dyson equations for the fermion self-energy using an appropriate truncation scheme. We analyzed two versions of the model: in the first case we considered the single fermion and in the second case we studied the version of the theory with $N$ fermion flavors within the large- $N$ expansion. In both cases we used the linearized equations. We have also used the rainbow-quenched approximations for the first case and rainbow-unquenched approximations to leading order in $1 / N$ in the second one.

In the rainbow-quenched approximation, when the continuum limit $(\Lambda \rightarrow \infty)$ for the massless theory is considered, we obtained a nontrivial analytical solution for the dynamical fermion mass for all values of the coupling constant, indicating the existence of CSB. For finite cutoff we obtained a nontrivial analytical solution for the mass function only for the regime in which $\alpha>\alpha_{c}=\pi / 4 \approx$ 0.78 , and so there is CSB. This mass function exhibits a phase transition as $\alpha \rightarrow \alpha_{c}$ and obeys the Miransky scaling law.

In the rainbow-unquenched approximation for $\Lambda \rightarrow \infty$, the CSB occurs for all values of the number of fermion flavors. For finite cutoff there is, however, a critical flavor number $N_{c}$, given by Eq. (31), below which there is CSB and above which the chiral symmetry is restored. For a large coupling this critical number is the same as for $\mathrm{QED}_{3}$, and the mass scale has a similar Miransky scaling law as within the quenched approximation. This is a result quite different from that obtained in $\mathrm{QED}_{4}$, where the inclusion of the fermion loop changes nontrivially the vacuum polarization, and thus changing the Miransky scaling law. This is due to the fact that the gauge-field propagator is analytic in $\mathrm{QED}_{4}$ and the fermion loop gives rise to its nontrivial renormalization while in $\mathrm{PQED}_{3}$ the gauge-field propagator is nonanalytic and because of that the kernel changes only trivially, i.e., by an additive factor.

In summary we would like to point out some important features of $\mathrm{PQED}_{3}$ studied in this paper. First, the gaugefield propagator is nonanalytic, and behaves as $1 / p$, as a function of the momentum. Furthermore, this theory exhibits an interesting critical behavior with chiral symmetry breaking resembling both $\mathrm{QED}_{3}$ and $\mathrm{QED}_{4}$. The same theory also describes two-dimensional condensed-matter systems with Dirac particles at low energies coupled with the gauge field propagating through three-dimensional space. In particular, the obtained results pertain to the description of the graphene system in the deep infrared regime where the Fermi velocity approaches the velocity of light. The same theory might also be relevant for the description of other condensed-matter systems featuring Dirac fermions, such as topological insulators [54].

Finally, the results of the present paper suggest that the retardation effects decrease the value of the critical coupling at which the dynamical mass generation occurs in the graphene system when contrasted with the case of a purely static Coulomb interaction [55-57]. However, to uncover the role of the retardation effects in determining the critical coupling in the experimentally accessible regime of the graphene physics, the starting point should be the theory (1) containing only the static Coulomb part of the full electromagnetic action, since $v_{F} \ll c$ in graphene. Including higher-order terms in $v_{F} / c$ will then determine the way retardation effects correct the value of the "bare" critical coupling, i.e., the critical coupling in the static (Coulomb) limit of the full electron-electron interaction. This investigation is in progress.

\section{ACKNOWLEDGMENTS}

We are grateful to E. C. Marino for stimulating discussions and encouragement and M. Gomes for helpful comments on SD equations. We would like to thank C. Morais Smith and B. Roy for critical reading of our manuscript and suggestions. We also thank D. Alves for his support and W. Lima for his help in numerical computations. V. S. A., L. O. N., and W. S. E. acknowledge financial support from CAPES-Brazil. V.S. A. and F. P. acknowledge Programa de Cooperación Internacional DI10-4002 of the Dirección de Investigación y Desarrollo de la Universidad de La Frontera (Temuco-Chile). V. J. acknowledges the support of the Netherlands Organization for Scientific Research (NWO). We would like to thank the Referee for his helpful comments and suggestions during the revision of this manuscript.

\section{APPENDIX A: NUMERICAL SOLUTION OF NONLINEAR INTEGRAL EQUATION}

In this appendix, we show the numerical solution of the integral equation for $\Sigma(p)$. The numerical solution was 
obtained by replacing the integral in Eq. (15) by the repeated trapezoidal quadrature rule [58], which transforms Eq. (15) (putting $m=0$ and $A(p)=1$ ) into a system of nonlinear algebraic equations

$$
\begin{aligned}
F_{m}\left(\left\{Z_{i}\right\}\right)= & \sum_{i=0}^{M} \delta_{m i} Z_{i}-\frac{\alpha}{2 \pi} \sum_{i=0}^{M-1} \frac{1}{2} h\left[f\left(y_{i}, x, Z_{i}\right)\right. \\
& \left.+f\left(y_{i+1}, x, Z_{i+1}\right)\right]=0,
\end{aligned}
$$

where the roots of the above equation are numerical solution for the mass function and

$$
f\left(y_{i}, x, Z_{i}\right)=\frac{y_{i} Z_{i}}{x\left(y_{i}^{2}+Z_{i}^{2}\right)}\left(\left|x+y_{i}\right|-\left|x-y_{i}\right|\right),
$$

with $m=0,1, \ldots, M$. Here, $\Sigma(p)$ is given by $Z_{i}, M$ is the number of intervals, $h$ is the size of each interval, and $y_{i}=0, \ldots, M$ are the mesh points.

In $\mathrm{PQED}_{3}$ quenched-rainbow approximation, the numerical solutions show that there is a critical value for the coupling constant close to the analytical result $\alpha_{c}=\pi / 4$; see Fig. 3. Setting $M=300$ as the number of intervals and choosing $10^{-3}<x, y_{i}<10$ (implying that $\Lambda=10)$, gives rise to the separation of points equal to $h=$ $\left(10-10^{-3}\right) /(300-1) \approx 0.033$. According to our results, the maximum of the mass function $\Sigma(p)$ (finite value), for all values of the coupling constant $\alpha$ is related to the minimum external momentum $p_{0}=10^{-3}$, i.e., $\Sigma\left(p_{0}\right) \geq$ $\Sigma(p)$. Furthermore, $\Sigma(\Lambda) \ll \Sigma\left(p_{0}\right)$ as expected of the integral equation. These conclusions are shown in Fig. 3, and in particular, the relation $\Sigma\left(p, \alpha>\alpha_{c}\right) \gg \Sigma(p, \alpha<$ $\alpha_{c}$ ) $\approx 0$ (in order $10^{-4}$ less than $p_{0}$ ) is obtained, allowing to interpret $\alpha_{c}$ as the critical coupling. General results, including the critical point, are independent of the cutoff $\Lambda$, but the values of $\Sigma(p)$ clearly change as $\Lambda$ varies. Here, for convenience, we choose $\Lambda=10$, i.e., we measure the momentum $p$ in units of $\Lambda / 10$, in order to provide the numerical results.

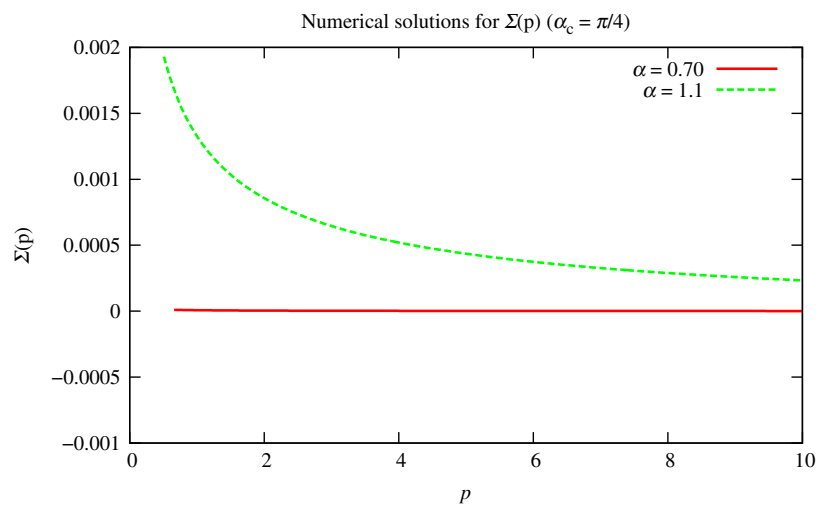

FIG. 3 (color online). Quenched-rainbow approximation in $\mathrm{PQED}_{3}$. Numerical solutions showing the momentum dependence of the dynamically generated fermion mass for $\alpha=0.70$ and $\alpha=1.1$. The number of mesh points is $M=300$.

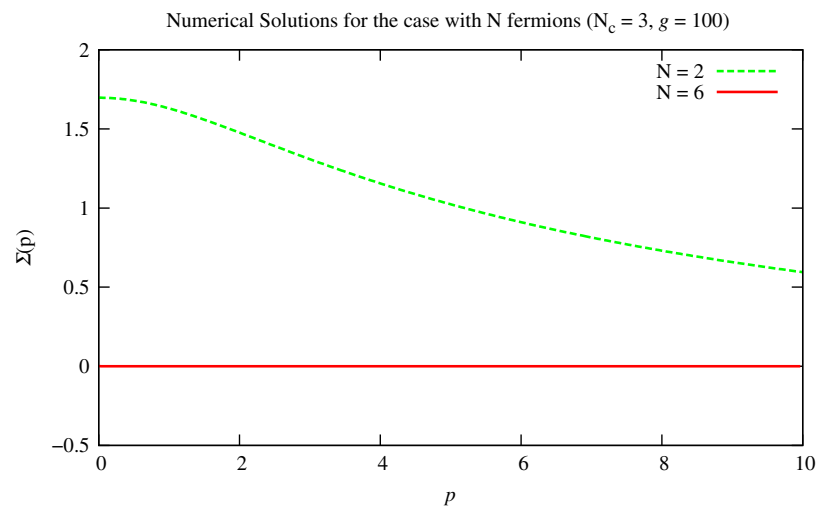

FIG. 4 (color online). Unquenched-rainbow approximation in $\mathrm{PQED}_{3}$. Numerical solutions showing the momentum dependence of the dynamically generated fermion mass for $N=2$ and $N=6$. The number of mesh points is $M=300$ and we choose for this case $g=100\left(N_{c}=3\right)$.

In $\mathrm{PQED}_{3}$ unquenched-rainbow approximation we have a new parameter $N$ (number of fermion flavors). There is a critical number, $N_{c}=\frac{4 g}{\pi^{2}\left(1+\frac{g}{8}\right)}$ and if we choose $N<N_{c}$, the numerical computation shows that there is a solution for $\Sigma(p)$ and so there is CSB. When we considered $N>N_{c}$ the only solution is $\Sigma(p) \approx 0$ (see Fig. 4). We assume finite cutoff in this computation. Figures 3 and 4 show us that the nonlinearity of integral equation (16) does not influence the CSB. These numerical results are consistent with the analytical results presented in the main text.

\section{APPENDIX B: THE WAVE FUNCTION RENORMALIZATION FUNCTION $A(p)$}

In this appendix, we show the numerical solution of the integral equation for $A(p)$, in the unquenched approximation and symmetric phase. We show that the wave function renormalization function is approximately one for all values of the external momentum when $\Sigma(p)=0$

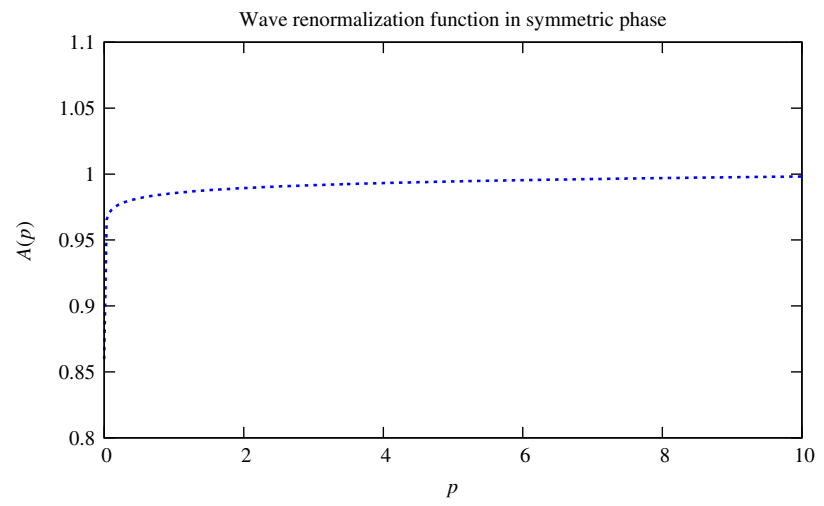

FIG. 5 (color online). Numerical results for the wave function renormalization function, $A(p)$, in the unquenched approximation and symmetric phase for $g=0.5$ and $N=1$. Notice that $A(p) \approx 1$, confirming the rainbow approach. 
(the symmetric phase). Multiplying the Eq. (13) by $\gamma^{\mu} p_{\mu}$ and taking the trace over the $\gamma$ matrices, we obtain

$A(p)=1+\frac{2 g}{N p^{2}} \int \frac{d^{3} k}{(2 \pi)^{3}} \frac{A(k) \Delta(q)}{k^{2} A^{2}(k)+\Sigma^{2}(k)} \frac{(p . q)(k . q)}{q^{2}}$,

(B1) where $q=p-k$ and

$$
\Delta(q)=\frac{1}{\left(q^{2}\right)^{1 / 2}(1+g / 8)} .
$$

We can exactly solve the angular integral in the above equation to obtain

$$
\begin{aligned}
A(p)= & 1-\frac{2 g}{N p^{3}} \frac{1}{16 \pi^{2}} \frac{1}{(1+g / 8)} \int_{0}^{\infty} d k \frac{k A(k)}{k^{2} A^{2}(k)+\Sigma^{2}(k)}\left\{\frac{1}{3}(p+k)^{3}-\frac{1}{3}|p-k|^{3}+\left(p^{2}-k^{2}\right)[(p-k) \operatorname{Sgn}(p+k)\right. \\
& -(p+k) \operatorname{Sgn}(p-k)]\},
\end{aligned}
$$

where $\operatorname{Sgn}(x)$ is the sign function. The integral equation above does not have an analytical solution as in $\mathrm{QED}_{3}$ and $\mathrm{QED}_{4}$. Furthermore, it explicitly depends on all values $\Sigma(k)$. At this point, we emphasize that we are interested in the study of the CSB and analyze the critical behavior of the theory. In order to do so, it is necessary to keep only the symmetric values of $A(p)$ and $\Pi(p)$, as extensively discussed in Ref. [12]. Taking $\Sigma^{2}(k)=0$ in the above integral equation, we obtain numerical values to $A(p)$ (see Fig. 5) showing that $A(p)=1$ is a good approximation for studying CSB in $\mathrm{PQED}_{3}$.
[1] T. Appelquist and R. D. Pisarski, Phys. Rev. D 23, 2305 (1981).

[2] R. D. Pisarski, Phys. Rev. D 29, 2423 (1984).

[3] T.W. Appelquist, Prog. Theor. Phys. Suppl. 85, 244 (1985).

[4] T. W. Appelquist, M. Bowick, D. Karabali, and L.C.R. Wijewardhana, Phys. Rev. D 33, 3704 (1986).

[5] T. W. Appelquist, D. Nash, and L.C.R. Wijewardhana, Phys. Rev. Lett. 60, 2575 (1988).

[6] G. W. Semenoff and L.C.R. Wijewardhana, Phys. Rev. Lett. 63, 2633 (1989).

[7] D. Nash, Phys. Rev. Lett. 62, 3024 (1989).

[8] C. J. Burden and C.D. Roberts, Phys. Rev. D 44, 540 (1991).

[9] C. J. Burden, J. Praschifka, and C. D. Roberts, Phys. Rev. D 46, 2695 (1992).

[10] P. Maris, Phys. Rev. D 52, 6087 (1995).

[11] G. Grignani, G. Semenoff, and P. Sodano, Phys. Rev. D 53, 7157 (1996).

[12] P. Maris, Phys. Rev. D 54, 4049 (1996).

[13] A. Bashir, A. Raya, I. C. Cloët, and C. D. Roberts, Phys. Rev. C 78, 055201 (2008).

[14] F. J. Dyson, Phys. Rev. 75, 1736 (1949).

[15] J. S. Schwinger, Proc. Natl. Acad. Sci. U.S.A. 37, 452 (1951); 37, 455 (1951).

[16] For an excellent review on SD equations, see C.D. Roberts and A. G. Williams, Prog. Part. Nucl. Phys. 33, 477 (1994).

[17] K. Johnson, M. Baker, and R. Wiley, Phys. Rev. 136, B1111 (1964); 163, 1699 (1967).

[18] T. Maskawa and H. Nakajima, Prog. Theor. Phys. 52, 1326 (1974); 54, 860 (1975).

[19] R. Fukuda and T. Kugo, Nucl. Phys. B117, 250 (1976).
[20] D. C. Curtis and M. R. Pennington, Phys. Rev. D 42, 4165 (1990).

[21] D. Atkinson, V.P. Gusynin, and P. Maris, Phys. Lett. B 303, 157 (1993).

[22] P. I. Fomin, V.P. Gusynin, V. A. Miransky, and Yu. A. Sitenko, Riv. Nuovo Cimento Soc. Ital. Fis. 6, 1 (1983); V. A. Miransky, Phys. Lett. 165B, 401 (1985); V. A. Miransky, Nuovo Cimento Soc. Ital. Fis. 90A, 149 (1985).

[23] J. B. Kogut, E. Dagotto, and A. Kocic, Phys. Rev. Lett. 60, 772 (1988); Nucl. Phys. B317, 253 (1989); E. Dagotto, J. B. Kogut, and A. Kocic, Phys. Rev. D 43, R1763 (1991).

[24] K. Kondo, Y. Kikukawa, and H. Mino, Phys. Lett. B 220, 270 (1989); V. P. Gusynin, Mod. Phys. Lett. A 05, 133 (1990).

[25] A. Bashir, C. Calcaneo-Roldan, L. X. Gutiérrez-Guerrero, and M.E. Tejeda-Yeomans, Phys. Rev. D 83, 033003 (2011).

[26] A. Kizilersu and M. R. Pennington, Phys. Rev. D 79, 125020 (2009).

[27] The value of the critical number of the fermion flavors $N_{c}=32 / \pi^{2}$ for the CSB can be changed when shortrange interactions are also considered in the $\mathrm{QED}_{3}$ theory, as shown in K. Kaveh and I. F. Herbut, Phys. Rev. B 71, 184519 (2005).

[28] E. Dagotto, J. B. Kogut, and A. Kocic, Phys. Rev. Lett. 62, 1083 (1989).

[29] A. Bashir and M. R. Pennington, Phys. Rev. D 50, 7679 (1994).

[30] A. Kizilersü, M. Reenders, and M. R. Pennington, Phys. Rev. D 52, 1242 (1995); C. J. Burden and P.C. Tjiang, Phys. Rev. D 58, 085019 (1998); A. Bashir, A. Kizilersü, and M. R. Pennington, Phys. Rev. D 62, 085002 (2000); A. Bashir and A. Raya, Phys. Rev. D 64, 105001 (2001); 
A. Bashir, A. Huet, and A. Raya, Phys. Rev. D 66, 025029 (2002); C. S. Fischer, R. Alkofer, T. Dahm, and P. Maris, Phys. Rev. D 70, 073007 (2004); A. Kizilersü and M. R. Pennington, Phys. Rev. D 79, 125020 (2009).

[31] M. R. Pennington and S. P. Webb, Brookhaven Nat. Lab. Report No. BNL-40886, 1988; D. Atkinson, P. Johnson, and M.R. Pennington, Brookhaven Nat. Lab. Report No. BNL-41615 (1988); D. Atkinson, P. W. Johnson, and P. Maris, Phys. Rev. D 42, 602 (1990); T. Matsuki, Z. Phys. C 51, 429 (1991); M. R. Pennington and D. Walsh, Phys. Lett. B 253, 246 (1991); D. C. Curtis, M. R. Pennington, and D. Walsh, Phys. Lett. B 295, 313 (1992).

[32] In fact, this result can be seen heuristically by considering the classical potential $V(\vec{r})=e^{2} \int_{-\infty}^{+\infty} d x_{3} \int\left(d^{3} k /(2 \pi)^{3}\right) \times$ $\left(e^{i\left(\vec{k} \cdot \vec{r}+k_{3} x_{3}\right)} /|k|\right)$, where $|k|=\sqrt{k^{2}}=\sqrt{\vec{k}^{2}+k_{3}^{2}}$. After carrying out the angular integral, we get $V(r)=\frac{e^{2}}{2 \pi} \times$ $\int_{0}^{\infty} J_{0}(|\vec{k}||\vec{r}|) d|\vec{k}|=\frac{e^{2}}{2 \pi} \frac{1}{|\vec{r}|}$. See formula 6.511 in I.S. Gradshteyn and I. M. Ryzhik, Table of Integrals, Series, and Products (Academic, New York, 1963).

[33] E. C. Marino, Nucl. Phys. B408, 551 (1993).

[34] J. González, F. Guinea, and M. A. H. Vozmediano, Nucl. Phys. B424, 595 (1994).

[35] R. L. P. G. do Amaral and E. C. Marino, J. Phys. A: Math. Gen. 25, 5183 (1992); D. G. Barci, L. E. Oxman, and M. Rocca, Int. J. Mod. Phys. A 11, 2111 (1996).

[36] F. J. Dyson, Phys. Rev. 75, 486 (1949).

[37] A.H. Castro Neto, F. Guinea, N. M. R. Peres, K. S. Novoselov, and A. K. Geim, Rev. Mod. Phys. 81, 109 (2009).

[38] S. Teber, Phys. Rev. D 86, 025005 (2012).

[39] B. Roy, V. Juričić, and I. F. Herbut, Phys. Rev. B 87, 041401(R) (2013).

[40] I. F. Herbut and V. Mastropietro, arXiv:1304.1988.

[41] G. W. Semenoff, Phys. Rev. Lett. 53, 2449 (1984).

[42] I. F. Herbut, V. Juričić, and B. Roy, Phys. Rev. B 79, 085116 (2009).

[43] S. Ryu, C. Mudry, C.-Y. Hou, and C. Chamon, Phys. Rev. B 80, 205319 (2009).
[44] B. Roy and I.F. Herbut, Phys. Rev. B 82, 035429 (2010).

[45] In a three-dimensional space-time we may also use the $2 \times 2$ representation for the $\gamma$ matrices. These could be the Pauli matrices $\gamma_{0}=i \sigma_{1}, \gamma_{1}=i \sigma_{2}$, and $\gamma_{2}=i \sigma_{3}$, which satisfy the algebra $\left\{\gamma_{\mu}, \gamma_{\nu}\right\}=-2 \delta_{\mu \nu}$. However, in this representation we cannot construct a fourth matrix that anticommutes with all others, and therefore no chiral symmetry operator exists in this representation. Notice that in this representation the generation of the mass term $m \bar{\psi} \psi$ breaks the parity symmetry. For more details, see Refs. [2,3,6].

[46] M. Gomes, R. S. Mendes, R. F. Ribeiro, and A. J. da Silva, Phys. Rev. D 43, 3516 (1991).

[47] We use that $\operatorname{Tr}\left(\gamma_{\mu} \gamma_{\nu}\right)=-4 \delta_{\mu \nu}, \operatorname{Tr}\left(\gamma^{\mu} \gamma^{\nu} \gamma^{\alpha}\right)=0$ and $\operatorname{Tr}\left(\gamma^{\mu} \gamma^{\nu} \gamma^{\alpha} \gamma^{\beta}\right)=4\left(\delta^{\mu \nu} \delta^{\alpha \beta}-\delta^{\mu \alpha} \delta^{\nu \beta}+\delta^{\mu \beta} \delta^{\nu \alpha}\right)$.

[48] P. Maris, Phys. Rev. D 50, 4189 (1994).

[49] T. Appelquist, M.J. Bowick, E. Cohler, and L.C. R. Wijewardhana, Phys. Rev. Lett. 55, 1715 (1985).

[50] P. I. Fomin, V. P. Gusynin, and V. A. Miransky, Phys. Lett. 78B, 136 (1978).

[51] D. Atkinson and P. W. Johnson, Phys. Rev. D 35, 1943 (1987).

[52] W. A. Bardeen, C. N. Leung, and S. T. Love, Phys. Rev. Lett. 56, 1230 (1986).

[53] I. F. Herbut, Phys. Rev. Lett. 87, 137004 (2001).

[54] M.Z. Hasan and C. L. Kane, Rev. Mod. Phys. 82, 3045 (2010); X.-L. Qi and S.-C. Zhang, Rev. Mod. Phys. 83, 1057 (2011).

[55] J. E. Drut and T. A. Lahde, Phys. Rev. Lett. 102, 026802 (2009).

[56] J.E. Drut and T. A. Lahde, Phys. Rev. B 79, 165425 (2009).

[57] C.-X. Zhang, G.-Z. Liu, and M.-Q. Huang, Phys. Rev. B 83, 115438 (2011).

[58] W. H. Press, S. A. Teukolsky, W. T. Vetterling, and B. P. Flannery, Numerical Recipes in Fortran 90: The Art of Parallel Scientific Computing (Cambridge University Press, Cambridge, England, 1996), Vol. 2. 